\title{
ESR1, HK3 and BRSK1 gene variants are associated with both age at natural menopause and premature ovarian failure
}

Yingying Qin ${ }^{1}$, Mei Sun ${ }^{1}$, Li You$^{1}$, Deying Wei ${ }^{2}$, Jielin Sun ${ }^{3}$, Xiaoyan Liang ${ }^{4}$, Bo Zhang ${ }^{5}$, Hong Jiang ${ }^{6}$, Jianfeng Xu ${ }^{7}$ and Zi-Jiang Chen ${ }^{1 *}$

\begin{abstract}
Background: Premature ovarian failure (POF) is a complex and heterogeneous disorder that is influenced by multiple genetic components. Numerous candidate gene studies designed to identify POF susceptibility loci have been published, but most positive findings have not been confirmed in follow up studies. We sought to determine if sequence variants previously associated with age at natural menopause (AANM) or early menopause (EM) contribute as well to genetic susceptibility to POF.

Methods: Our study was performed on 371 unrelated idiopathic women with POF and 800 women controls, all Chinese Han. Thirty six SNPS from previous genome-wide association studies (GWAS) responsible for AANM or EM and 3 additional SNPs in ESR1, and 2 additional SNPs in PTHB1 were tested using the Sequenom MassARRAY iPLEX platform for genotyping.

Results: Three SNPs - rs2278493 in HK3, rs2234693 in ESR1 and rs12611091 in BRSK1 - showed nominally significant association with POF. Thus, a plausible relationship could exist between ESR1, BRSK1, HK3 and POF.

Conclusions: This largest association study undertaken to determine correlation between POF and AANM/EM revealed three significant SNPs (rs2278493, rs2234693, and rs12611091). All are associated with not only AAWM and EM but also POF. Insights into shared genetic susceptibility between POF and AANM/EM will provide novel entry points for unraveling genetic mechanism involved in ovarian reserve and oocyte aging processes.
\end{abstract}

Keywords: Premature ovarian failure (POF), Primary ovarian insufficiency (POI), Age at natural menopause (AANM), HK3, ESR1, BRSK1, Premature menopause, Hypergonadotropic hypogonadism

\section{Background}

Natural menopause is one of the most important physiological events, the cessation of ovarian function and the end of the reproductive lifespan. Twin and family studies indicate high heritability for age at natural menopause (AANM) (49-87\%) [1]. The AANM in Chinese women is approximately 49 years [2]. Early onset of menopause increases risk for many postmenopausal health complications. Menopause occurring between 40 and 44 years has

\footnotetext{
* Correspondence: chenzijiang@hotmail.com

${ }^{1}$ Center for Reproductive Medicine, Provincial Hospital Affiliated to Shandong University, National Research Center for Assisted Reproductive Technology and Reproductive Genetics, The Key laboratory for Reproductive Endocrinology of Ministry of Education, Shandong Provincial Key Laboratory of Reproductive Medicine, Jinan, China

Full list of author information is available at the end of the article
}

an incidence of $5 \%$ and is termed early menopause (EM) [3]. Premature ovarian failure (POF), also termed as primary ovarian insufficiency (POI), is defined as ovarian failure before the age 40 years $[4,5]$.

POF is characterized by secondary amenorrhea, hypoestrogenism and elevated gonadotropin serum levels (FSH > $40 \mathrm{IU} / \mathrm{L})$. Approximately $1 \%$ of the population has POF before age 40 years; only $0.1 \%$ or less are affected prior to age 30. Although most cases of POF occur sporadically, approximately $10-15 \%$ of cases have an affected firstdegree relative [6]. Based on either known roles in folliculogenesis or phenotypes of murine knockout models, many candidate genes have been interrogated to determine their role in POF. However, except for FSHR in Finnish women none explains more than $1-2 \%$ of POF in 
any given ethnic group [7]. Genes whose perturbations cause POF have nonetheless been identified in single patients or families, small patient groups or selected ethnic groups. This may reflect genetic heterogeneity, ethnic-specific genetic variation or failure to interrogate sufficient numbers of the candidate genes that could potentially account for POF.

Via genome-wide association studies (GWASs), it is possible to identify common genetic variants contributing to susceptibility to genetically complex or polygenic diseases. Despite the attractiveness of GWAS for POF, genome searches in a sufficiently large number of patients are practically absent [8]. Kang H.J. et al. showed association of two SNPs (rs3884597 and rs6944723) in the PTHresponsive B1 gene (PTHB1) in a small discovery set of 24 women and 24 controls, followed by confirmation in 101 cases and 87 controls [9]. The other GWAS showed association with ADAMTS19 in the discovery set of 99 Dutch women and 181 controls, but not in the replication set (60 POF cases and 90 controls) [10]. That $8 \%$ of the relatives of women with POF in Chinese suffer from either early menopause or POF (Table 1) suggests an interrelated genetic mechanism between POF and early menopause. Indeed, Tibiletti et al. proposed that POF and EM patients share similar genetic features and postulated that these conditions may be a variable expression of the same genetic disease [11]. Additionally, FMR1 is a single gene cause of POF, and it is clinically indicated to test for premutation in this gene. Genes like EIF2B5 and FMR1 that are known to be involved in POF have been reported to be associated also with AANM [12-14]. Few studies have explored the shared genetic associations among POF, AANM and EM. On the basis of four independent GWAS, 36 SNPs have been associated with AANM. These are located on chromosomes $2,3,5,6,7,8,9,11,12,13$, $15,18,19$ and 20, respectively, with robust statistical evidence. Of these, 2 SNPs were related to EM $[1,15,16]$. Besides these 36 SNPs, we also assessed correlation between POF and three additional SNPs in the estrogen receptor 1 (ESR1) gene, given the key role of ER- $\alpha$ in folliculogenesis. ESR1 has also been considered a promising candidate gene for POF, with rs2234693, rs9340799 and rs1569788 of ESR1 reported to be associated with idiopathic POF [17-19]. ESR1 also has been one of the most popular candidates related with AANM in previous association studies [20,21].

Overall, we sought to determine the contribution to POF of the 36 SNPs from AANM GWAS, the 3 SNPs in the ESR1 gene and the 2 SNPs in the PTHB1 gene. Our association study involved 371 women with POF and 800 controls, all Chinese Han.

\section{Methods}

\section{Participants and phenotypes}

Our study was performed on 371 unrelated idiopathic women with POF and 800 women controls, all Chinese Han, collectively gathered from a number of high volume regional referral centers in China. Samples were collected from 18 provinces during 2002-2010. The age of secondary amenorrhea in our sample was young

Table 1 Clinical features of cases and controls, which are not significantly different $(p>0.05)$.

\begin{tabular}{|c|c|}
\hline Characteristic & \\
\hline \multicolumn{2}{|l|}{ Cases } \\
\hline Number & 371 \\
\hline FSH concentration $(\mathrm{IU} / \mathrm{L})^{\mathrm{a}}$ & $73.88 \pm 30.23$ \\
\hline Familial clustering ${ }^{\mathrm{b}}$ & 32 \\
\hline Mother affected & 27 \\
\hline Menopause $<40$ yrs & 13 \\
\hline 40 yrs $<$ Menopause $<45$ yrs & 14 \\
\hline Siblings affected & 5 \\
\hline Menopause $<40$ yrs & 5 \\
\hline Age at diagnosis $(y r s)^{a}$ & $29.79 \pm 5.31$ \\
\hline Amenorrhea Secondary & 228 \\
\hline Age at menarche (yrs) ${ }^{a}$ & $14.82 \pm 2.11$ \\
\hline Age at onset of menstrual dysfunction (yrs) ${ }^{a}$ & $23.85 \pm 6.78$ \\
\hline Age at amenorrhea $(y r s)^{a}$ & $24.87 \pm 5.88$ \\
\hline \multicolumn{2}{|l|}{ Controls } \\
\hline Number & 800 \\
\hline Age $(y r s)^{a}$ at sample & $38.77 \pm 11.58$ \\
\hline Age at menarche(yrs) ${ }^{a}$ & $14.75 \pm 1.45$ \\
\hline
\end{tabular}


(mean age $23.85 \pm 6.78$ years) (Table 1). 8\% (32/400) of cases had a positive family history, i.e., at least two firstor second-degree female family members with POF or early menopause including the index POF patient. Most of the affected relatives (27/32; thirteen $<40$ yrs and fourteen $<45 \mathrm{yrs}$ ) were mothers of the index patient; the remaining five were sisters. Inclusion criteria for POF consisted of cessation of menstrual cycles before 40 years of age, with at least two serum follicle stimulating hormone (FSH) concentrations exceeding $40 \mathrm{IU} / \mathrm{L}$. Serum FSH level was determined by the electrochemiluminescent immunoassay in the analyzer cobas e 601 (Roche, Switzerland). The analytical sensitivity of basal FSH was $<0.10 \mathrm{mIU} / \mathrm{ml}$. All samples were run in the same assay. Early menopause is defined as menopause that occurs between ages 40 and 44 . Women with known chromosomal abnormalities, previous ovarian surgery, chemotherapy, or radiotherapy were excluded. Controls attended the clinic because of tubal factor infertility. They were known to be menstruating regularly and confirmed by hormone assays $(<40 \mathrm{mIU} / \mathrm{L})$ and ultrasound imaging to be free of POF-related and early menopause- related phenotypes. Informed consent was obtained from all subjects. The study was approved by the Institutional Review Board of Reproductive Medicine of Shandong University.

\section{Genotyping and statistical analysis}

SNPs were chosen for genotyping on the basis of published findings in association studies. A total of 41 SNPs are located in LHCGR, PPARG, SRD5A1, HK3, RAP80, SYCP2L, TNF, ESR1, IGF2R, PTHB1, NBN, TGFBR1, FSHB, PGR, ANKK1, IGF1, CYP19A1, POLG, SMAD7, BRSK1, TMEM150B, MCM8 or intergenic region (Table 2). We used the Sequenom MassARRAY iPLEX platform for genotyping. The Sequenom protocol involves a multiplex PCR reaction prior to a single-base primer extension reaction. The individual SNPs are identified by using matrixassisted laser desorption/ionization time- of- fight mass spectrometry. SNPs were excluded if the minor allele frequency was $<1 \%$. Ultimately, 36 SNPs were genotyped in the case control cohort. Allelic association analysis was conducted using SHEsis [22]. The genotype distributions for all SNPs were tested for Hardy-Weinberg equilibrium (HWE) using the Fisher's exact test. All SNPs were in Hardy- Weinberg equilibrium. The allelic odds ratio (OR) and 95\% confidence interval (CI) were estimated assuming a multiplicative model. P-values were two-tailed. An alpha of 0.05 was used to claim statistical significance.

\section{Results}

We analyzed 41 SNPs for association with POF in our collection of 400 cases and 800 controls. The analysis was nominally significant for three SNPs: rs2278493 in
HK3, rs2234693 in ESR1 and rs12611091 in BRSK1, respectively. Results are summarized in Table 2 . These findings demonstrate that certain SNPs associated with AANM also may contribute to POF, but need not necessarily play a major role in POF. No significant association with POF was observed for the remaining 38 SNPs.

\section{Discussion}

We conducted the largest association study reported to date in a POF cohort in order to test the contribution to POF of 36 SNPs, specifically known to be associated with AANM and EM. We also investigated the associations of 3 ESR1 SNPs and 2 PTHB1 SNPs in POF. We found contribution to POF for SNPs in ESR1, BRSK1 and HK3, 2 of these genes not previously considered as candidates for POF.

Estrogen regulates cyclic gonadotropin release at the hypothalamus- hypophysis- ovarian (HPO) axis by the estrogen receptor $\alpha$ (ER- $\alpha)$, which is encoded by ESR1 and enhances follicular development by the ER- $\beta$ encoded by ESR2. Female ER- $\alpha$ knockout mice show anovulation and complete infertility. Rs2234693 lies in an intron of the ESR1gene and has been reported to be related to POF and the onset of natural menopause in Korean and Dutch [17-19], consistent with our results. Because this SNP does not change any amino acid in the ERS1 protein, it is presumed to be in linkage disequilibrium with a regulatory sequence(s) that could affect gene expression or function and consequently result in an alteration in estrogenic biological activity.

Rs12611091 is located in the intron region of BRSK1 (BR serine/threonine kinase 1), a gene that codes for an AMP-activated protein kinase (AMPK)-related kinase [23]. BRSK1 is highly expressed in human brain and moderately expressed in mammalian ovaries [24]. Mutation or overexpression of the BRSK1 gene deleteriously affects vesicle transport and release at the axon terminals [25]. BRSK1 might affect the secretion of gonadotropin- releasing hormone $(\mathrm{GnRH})$ from the hypothalamus through this mechanism. Additionally, maternal embryonic leucine zipper kinase (MELK), which is highly expressed in spermatogonia and oocytes, is one of the downstream targets of BRSK1 $[26,27]$. Plausible involvement in ovarian aging is therefore suggested for BRSK1.

Hexokinase 3 (white cell) (HK3), one of the four hexokinase family members, is involved in carbohydrate metabolism through phosphorylating glucose to produce glucose-6-phosphate [28]. HK3 is present in uterus, placenta, lung and adipose according to GeneAtlas [24]. Literature analysis for HK3 did not indicate an immediate functional explanation for the observed association. To elucidate the precise molecular mechanism, extensive sequence analysis and functional studies of the HK3gene variants are needed. 
Table 2 Loci selected for association analysis in POF and controls.

\begin{tabular}{|c|c|c|c|c|c|c|c|c|c|c|c|c|}
\hline \multicolumn{11}{|c|}{ All samples ( 371 cases and 800 controls) } & \multirow[t]{2}{*}{ Gene information } & \multirow[t]{2}{*}{ Phenotype related } \\
\hline $\mathrm{CHR}$ & BP & SNP & F_MISS & HWE_P & A1 & F_A & F_U & A2 & $\mathbf{P}$ & OR & & \\
\hline 3 & 12423994 & rs4135280 & 0.008333 & 0.2789 & C & 0.2861 & 0.2738 & $\mathrm{~T}$ & 0.5321 & 1.063 & PPARG & AANM \\
\hline 5 & 6692179 & rs494958 & 0.009167 & 0.3597 & $\mathrm{~T}$ & 0.2263 & 0.2237 & A & 0.8881 & 1.015 & SRD5A1 & AANM \\
\hline 5 & 176247040 & rs2278493 & 0.008333 & 0.08305 & A & 0.3451 & 0.3881 & G & 0.0432 & 0.8309 & HK3 & AANM \\
\hline 5 & 176255904 & rs691141 & 0.0175 & 0.682 & $\mathrm{~T}$ & 0.4747 & 0.4608 & $C$ & 0.5302 & 1.057 & HK3 & AANM \\
\hline 5 & 176290671 & rs7718874 & 0.01083 & 0.4156 & $\mathrm{~T}$ & 0.4696 & 0.4765 & $C$ & 0.7525 & 0.9726 & RAP80 & AANM \\
\hline 5 & 176311180 & rs365132 & 0.0175 & 0.4133 & $C$ & 0.4669 & 0.4744 & A & 0.7343 & 0.9704 & RAP80 & AANM \\
\hline 5 & 176367046 & rs402511 & 0.01833 & 0.293 & $C$ & 0.4707 & 0.4769 & $\mathrm{~T}$ & 0.7792 & 0.9755 & RAP80 & AANM \\
\hline 5 & 176436169 & rs244715 & 0.01083 & 0.3229 & A & 0.4842 & 0.4703 & G & 0.5254 & 1.058 & intergenic & EM \\
\hline 6 & 11005474 & rs2153157 & 0.0275 & 0.5569 & G & 0.3484 & 0.335 & A & 0.5233 & 1.061 & SYCP2L & AANM \\
\hline 6 & 31648292 & rs909253 & 0.01333 & 0.0382 & $C$ & 0.4134 & 0.3829 & $\mathrm{~T}$ & 0.1564 & 1.136 & TNF & AANM \\
\hline 6 & 152205028 & rs2234693 & 0.01 & 0.7176 & $C$ & 0.3655 & 0.4217 & $\mathrm{~T}$ & 0.009057 & 0.79 & ESR1 & POF/AANM \\
\hline 6 & 152205074 & rs9340799 & 0.006667 & 0.7148 & G & 0.189 & 0.2016 & A & 0.4703 & 0.9228 & ESR1 & POF/AANM \\
\hline 6 & 152370309 & rs1569788 & 0.008333 & 0.7268 & $\mathrm{~T}$ & 0.453 & 0.474 & $C$ & 0.338 & 0.9191 & ESR1 & POF/AANM \\
\hline 6 & 160419555 & rs2297362 & 0.008333 & 0.9062 & $C$ & 0.4301 & 0.4383 & $\mathrm{~T}$ & 0.7047 & 0.9669 & IGF2R & AANM \\
\hline 6 & 160423646 & rs9457827 & 0.01417 & 0.9059 & $\mathrm{~T}$ & 0.4312 & 0.4379 & $C$ & 0.7602 & 0.9732 & IGF2R & AANM \\
\hline 7 & 33351787 & rs3884597 & 0.009167 & 0.222 & $\mathrm{~T}$ & 0.467 & 0.4784 & G & 0.6047 & 0.9554 & PTHB1 & POF \\
\hline 7 & 33390485 & rs6944723 & 0.01083 & 1 & $A$ & 0.4459 & 0.4183 & $T$ & 0.2051 & 1.119 & PTHB1 & POF \\
\hline 8 & 91019056 & rs2697679 & 0.009167 & 0.5546 & G & 0.4195 & 0.437 & $\mathrm{~T}$ & 0.4217 & 0.931 & NBN & AANM \\
\hline 8 & 91082415 & rs7011299 & 0.015 & 0.5509 & G & 0.4237 & 0.4202 & $C$ & 0.8727 & 1.014 & NBN & AANM \\
\hline 9 & 100955986 & rs1590 & 0.0075 & 0.1803 & G & 0.4581 & 0.466 & $\mathrm{~T}$ & 0.7184 & 0.9687 & TGFBR1 & AANM \\
\hline 11 & 30196754 & rs11031010 & 0.0125 & 0.4482 & A & 0.04319 & 0.04047 & $C$ & 0.7559 & 1.07 & FSHB & AANM \\
\hline 11 & 112786283 & rs6279 & 0.0075 & 0.05507 & $C$ & 0.4619 & 0.4747 & G & 0.5609 & 0.9501 & ANKK1 & AANM \\
\hline 13 & 111017433 & rs1361542 & 0.005833 & 0.396 & A & 0.03665 & 0.03391 & G & 0.7332 & 1.084 & intergenic & AANM \\
\hline 13 & 111017854 & rs1756091 & 0.006667 & 0.3965 & A & 0.03655 & 0.03461 & $C$ & 0.8101 & 1.058 & intergenic & AANM \\
\hline 13 & 111019298 & rs7333181 & 0.0075 & 0.3965 & A & 0.03655 & 0.03465 & G & 0.8143 & 1.057 & intergenic & AANM \\
\hline 13 & 111019632 & rs1163623 & 0.003333 & 0.3965 & $C$ & 0.03627 & 0.03457 & $\mathrm{~T}$ & 0.8326 & 1.051 & intergenic & AANM \\
\hline 15 & 49335997 & rs11856927 & 0.005833 & 0.6833 & $C$ & 0.4532 & 0.4641 & A & 0.6187 & 0.9572 & CYP19A1 & AANM \\
\hline 15 & 87670333 & rs2351002 & 0.01083 & 0.5205 & G & 0.3521 & 0.3398 & $C$ & 0.5542 & 1.056 & POLG & AANM \\
\hline 18 & 44721958 & rs4939833 & 0.0075 & 0.1067 & G & 0.03403 & 0.04203 & A & 0.3495 & 0.803 & SMAD7 & AANM \\
\hline 19 & 60492141 & rs12611091 & 0.005833 & 0.8633 & $\mathrm{~T}$ & 0.2422 & 0.2001 & $C$ & 0.01892 & 1.278 & BRSK1 & AANM \\
\hline 19 & 60506693 & rs1551562 & 0.01917 & 0.161 & G & 0.06016 & 0.06974 & A & 0.3858 & 0.8539 & BRSK1 & AANM \\
\hline 19 & 60511657 & rs1172822 & 0.008333 & 0.384 & $\mathrm{~T}$ & 0.0953 & 0.09046 & $C$ & 0.7026 & 1.059 & BRSK1 & AANM \\
\hline 19 & 60516144 & rs7246479 & 0.0075 & 0.4537 & $C$ & 0.1929 & 0.1895 & A & 0.8435 & 1.022 & BRSK1 & AANM \\
\hline 19 & 60523000 & rs2384687 & 0.006667 & 0.4769 & $C$ & 0.08877 & 0.09023 & $\mathrm{~T}$ & 0.9072 & 0.9822 & TMEM150B & AANM \\
\hline 19 & 60525476 & rs11668344 & 0.005833 & 0.4762 & $C$ & 0.08724 & 0.08962 & $\mathrm{~T}$ & 0.8488 & 0.9709 & TMEM150B & AANM \\
\hline 19 & 60525566 & rs897798 & 0.0075 & 0.6029 & $C$ & 0.08924 & 0.09383 & $\mathrm{~T}$ & 0.7183 & 0.9463 & TMEM150B & AANM \\
\hline 11 & 30203352 & rs621686 & 0.01417 & 1 & G & 0.002653 & 0.00062 & $A$ & 0.1956 & 4.285 & FSHB & AANM \\
\hline 11 & 30218544 & rs7951733 & 0.003333 & 1 & G & 0 & 0.000617 & $A$ & 0.4899 & 0 & FSHB & AANM \\
\hline 11 & 100477546 & rs619487 & 0.003333 & 1 & $C$ & 0.003886 & 0.006173 & A & 0.4769 & 0.6281 & PGR & AANM \\
\hline 12 & 101388555 & rs1019731 & 0.0075 & 1 & $\mathrm{~T}$ & 0.002611 & 0.003094 & G & 0.8388 & 0.8435 & IGF1 & AANM \\
\hline 20 & 5896227 & rs16991615 & 0.01167 & 1 & $A$ & 0.001316 & 0.001241 & G & 0.9617 & 1.061 & MCM8 & EM \\
\hline
\end{tabular}

CRH: Chromosome; SNP: Single-nucleotide polymorphism; HWE_P: Hardy-Weinberg principle; F_A, F_U indicate the allele frequency in cases and controls, respectively

Idiopathic POF and EM differ in age of menopause onset and have long been considered to represent variable expression of the same genetic disease [11]. It is highly plausible that certain genes might contribute to both. That is, similar underlying genetic predisposition exists, possibly with different environmental factors or modifiers triggering disease progression and, hence, specific age of onset. Another possibility is that certain genes in pathways ordinarily predisposing only to EM interact or converge to cause even earlier age of onset, i.e, below age 40 and thus lead to clinical POF. 


\section{Conclusions}

In summary, our data demonstrate that genetic susceptibility for both AANM/EM and POF is shared by the ESR1, HK3 and BRSK1 genes. This became evident by our showing that three SNPs previously related to AANM were associated with POF. At these newly identified loci fine mapping or sequencing might lead to identification of causal variants, and thus expand our knowledge of the underlying mechanism of POF. Insights into shared genetic susceptibility between POF and AANM/EM will further provide novel entry points for unraveling etiology involved in ovarian reserve and aging process. Identifying genetic causation may further be of diagnostic value to other family members, especially female offspring. If a younger relative has the same genetic variant, timeline for achieving pregnancy might be advanced and early child-bearing encouraged.

\section{List of abbreviations}

AANM: age at natural menopause; EM: early menopause; POF: premature ovarian failure; POI: primary ovarian insufficiency; GWAS: genome-wide association study; PTHB1: PTH-responsive B1 gene; ESR1: estrogen receptor 1 FSH: follicle stimulating hormone; SNP: single nucleotide polymorphism; HPO: hypothalamus- hypophysis- ovarian; ER-a:estrogen receptor a; AMPK: AMP-activated protein kinase; MELK: maternal embryonic leucine zipper kinase; BRSK1: BR serine/threonine kinase 1; GnRH: gonadotropin- releasing hormone; HK3: Hexokinase 3 (white cell).

\section{Acknowledgements}

We thank all the participants involved in this study. Supported by grants from the National Basic Research Program of China (973 Program2012CB944700, 2010CB945002, 2007CB947403, 2007CB948103), A Foundation for the Author of National Excellent Doctoral Dissertation of PR China (201078), the National Natural Science Foundation of China (30973170, $81000236,81000238)$, Science research foundation item of no-earnings health vocation from Ministry of Public Health (201002013).

\section{Author details \\ ${ }^{1}$ Center for Reproductive Medicine, Provincial Hospital Affiliated to Shandong University, National Research Center for Assisted Reproductive Technology and Reproductive Genetics, The Key laboratory for Reproductive Endocrinology of Ministry of Education, Shandong Provincial Key Laboratory of Reproductive Medicine, Jinan, China. ${ }^{2}$ Department of Obstetrics and Gynecology, Provincial Hospital Affiliated to Shandong University, Jinan, China. ${ }^{3}$ Center for Cancer Genomics, Wake Forest University School of Medicine, Winston-Salem, North Carolina, USA. ${ }^{4}$ Center for Reproductive Medicine, the Sixth Affiliated Hospital of Sun Yat-Sen University, Guangzhou, China. ${ }^{5}$ Center for Reproductive Medicine, Maternal and Child Health Hospital in Guangxi, Guangxi, China. ${ }^{6}$ Center for Reproductive Medicine, 105th Hospital of PLA, Anhui, China. ${ }^{7}$ Fudan-VARI Center for Genetic Epidemiology, Fudan University, Shanghai, China.}

\section{Authors' contributions}

ZJC critically revised the manuscript, contributed to the study design analysis, and supervised the research. YYQ drafted and critically revised the manuscript, performed the statistical analyses, and contributed to the interpretation of the data. MS, DYW, XYL, BZ and HJ coordinated clinic diagnosis, the blood collection and storage of cases and controls. YL and JLS performed genotyping and statistical analyses. JFX contributed to the bioinformatics and the interpretation of the data. All authors read and approved the final manuscript.

\section{Competing interests}

The authors declare that they have no competing interests.
Received: 13 October 2011 Accepted: 17 January 2012

Published: 17 January 2012

\section{References}

1. Stolk L, Zhai G, van Meurs JB, Verbiest MM, Visser JA, Estrada K, Rivadeneira F, Williams FM, Cherkas L, Deloukas P, Soranzo N, de Keyzer JJ, Pop VJ, Lips P, Lebrun CE, van der Schouw YT, Grobbee DE, Witteman J, Hofman A, Pols HA, Laven JS, Spector TD, Uitterlinden AG: Loci at chromosomes 13, 19 and 20 influence age at natural menopause. Nat Genet 2009, 41:645-647.

2. Nie GN, Wang XY, Yang HY, Aihua Ou: The investigation and analysis of the factors related with the menopausal age of urban women in China. China maternal and child healthcare (Chinsese) 2011, 8:1191-1193.

3. Chang SH, Kim CS, Lee KS, Kim H, Yim SV, Lim YJ, Park SK: Premenopausal factors influencing premature ovarian failure and early menopause. Maturitas 2007, 58:19-30.

4. De Vos M, Devroey P, Fauser BC: Primary ovarian insufficiency. Lancet 2010, 376:911-921

5. Nelson LM: Clinical practice. Primary ovarian insufficiency. N Engl J Med 2009, 360:606-614

6. Simpson JL: Genetic and phenotypic heterogeneity in ovarian failure: overview of selected candidate genes. Ann N Y Acad Sci 2008, 1135:146-154.

7. Aittomaki K, Lucena JL, Pakarinen P, Sistonen P, Tapanainen J, Gromoll J, Kaskikari R, Sankila EM, Lehvaslaiho H, Engel AR, Nieschlag E, Huhtaniemi I, de la Chapelle A: Mutation in the follicle-stimulating hormone receptor gene causes hereditary hypergonadotropic ovarian failure. Cell 1995, 82:959-968.

8. Christin-Maitre S, Tachdjian G: Genome-wide association study and premature ovarian failure. Ann Endocrinol (Paris) 2010, 71:218-221.

9. Kang H, Lee SK, Kim MH, Song J, Bae SJ, Kim NK, Lee SH, Kwack K: Parathyroid hormone-responsive B1 gene is associated with premature ovarian failure. Hum Reprod 2008, 23:1457-1465.

10. Knauff EA, Franke $L$, van Es MA, van den Berg $L H$, van der Schouw $Y T$, Laven JS, Lambalk CB, Hoek A, Goverde AJ, Christin-Maitre S, Hsueh AJ, Wijmenga C, Fauser BC: Genome-wide association study in premature ovarian failure patients suggests ADAMTS19 as a possible candidate gene. Hum Reprod 2009, 24:2372-2378.

11. Tibiletti MG, Testa G, Vegetti W, Alagna F, Taborelli M, Dalpra L, Bolis PF, Crosignani PG: The idiopathic forms of premature menopause and early menopause show the same genetic pattern. Hum Reprod 1999, 14:2731-2734.

12. Fogli A, Gauthier-Barichard F, Schiffmann R, Vanderhoof VH, Bakalov VK, Nelson LM, Boespflug-Tanguy O: Screening for known mutations in EIF2B genes in a large panel of patients with premature ovarian failure. $B M C$ Womens Health 2004, 4:8.

13. Conway GS: Premature ovarian failure and FMR1 gene mutations: an update. Ann Endocrinol (Paris) 2010, 71:215-217.

14. He C, Kraft P, Chasman DI, Buring JE, Chen C, Hankinson SE, Pare G, Chanock S, Ridker PM, Hunter DJ: A large-scale candidate gene association study of age at menarche and age at natural menopause. Hum Genet 2010, 128:515-527.

15. Murray A, Bennett CE, Perry JR, Weedon MN, Jacobs PA, Morris DH, Orr N, Schoemaker MJ, Jones M, Ashworth A, Swerdlow AJ: Common genetic variants are significant risk factors for early menopause: results from the Breakthrough Generations Study. Hum Mol Genet 2011, 20:186-192.

16. He C, Kraft P, Chen C, Buring JE, Pare G, Hankinson SE, Chanock SJ, Ridker PM, Hunter DJ, Chasman DI: Genome-wide association studies identify loci associated with age at menarche and age at natural menopause. Nat Genet 2009, 41:724-728.

17. Kim S, Pyun JA, Cha DH, Ko JJ, Kwack K: Epistasis between FSHR and CYP19A1 polymorphisms is associated with premature ovarian failure. Fertil Steril 2011.

18. Yoon SH, Choi YM, Hong MA, Lee GH, Kim JJ, Im HJ, Min EG, Kang BM, Yoon BK, Moon SY: Estrogen receptor \{alpha\} gene polymorphisms in patients with idiopathic premature ovarian failure. Hum Reprod 2010, 25:283-287.

19. Bretherick KL, Hanna CW, Currie LM, Fluker MR, Hammond GL, Robinson WP: Estrogen receptor alpha gene polymorphisms are associated with idiopathic premature ovarian failure. Fertil Steril 2008, 89:318-324. 
20. Yang JJ, Cho LY, Lim YJ, Ko KP, Lee KS, Kim H, Yim SV, Chang SH, Park SK: Estrogen receptor-1 genetic polymorphisms for the risk of premature ovarian failure and early menopause. J Womens Health (Larchmt) 2010, 19:297-304.

21. Weel AE, Uitterlinden AG, Westendorp IC, Burger H, Schuit SC, Hofman A, Helmerhorst TJ, van Leeuwen JP, Pols HA: Estrogen receptor polymorphism predicts the onset of natural and surgical menopause. $J$ Clin Endocrinol Metab 1999, 84:3146-3150.

22. Shi YY, He L: SHEsis, a powerful software platform for analyses of linkage disequilibrium, haplotype construction, and genetic association at polymorphism loci. Cell Res 2005, 15:97-98.

23. Barnes AP, Lilley BN, Pan YA, Plummer LJ, Powell AW, Raines AN, Sanes JR, Polleux F: LKB1 and SAD kinases define a pathway required for the polarization of cortical neurons. Cell 2007, 129:549-563.

24. GeneAtlas. [http://genatlas.medecine.univ-paris5.fr/].

25. Lu R, Niida H, Nakanishi M: Human SAD1 kinase is involved in UVinduced DNA damage checkPOFnt function. I Biol Chem 2004, 279:31164-31170.

26. Kishi M, Pan YA, Crump JG, Sanes JR: Mammalian SAD kinases are required for neuronal polarization. Science 2005, 307:929-932.

27. Thelie A, Papillier P, Pennetier S, Perreau C, Traverso JM, Uzbekova S, Mermillod P, Joly C, Humblot P, Dalbies-Tran R: Differential regulation of abundance and deadenylation of maternal transcripts during bovine oocyte maturation in vitro and in vivo. BMC Dev Biol 2007, 7:125.

28. Nakamura N, Shibata H, O'Brien DA, Mori C, Eddy EM: Spermatogenic cellspecific type 1 hexokinase is the predominant hexokinase in sperm. Mol Reprod Dev 2008, 75:632-640.

doi:10.1186/1750-1172-7-5

Cite this article as: Qin et al.: ESR1, HK3 and BRSK1 gene variants are associated with both age at natural menopause and premature ovarian failure. Orphanet Journal of Rare Diseases 2012 7:5.

\section{Submit your next manuscript to BioMed Central and take full advantage of:}

- Convenient online submission

- Thorough peer review

- No space constraints or color figure charges

- Immediate publication on acceptance

- Inclusion in PubMed, CAS, Scopus and Google Scholar

- Research which is freely available for redistribution

Submit your manuscript at www.biomedcentral.com/submit 